\title{
When to Use Hierarchical Linear Modeling
}

\author{
Veronika Huta \\ a School of psychology, University of Ottawa
}

\begin{abstract}
Previous publications on hierarchical linear modeling (HLM) have provided guidance on how to perform the analysis, yet there is relatively little information on two questions that arise even before analysis: Does HLM apply to one's data and research question? And if it does apply, how does one choose between HLM and other methods sometimes used in these circumstances, including multiple regression, repeated-measures or mixed ANOVA, and structural equation modeling or path analysis? The purpose of this tutorial is to briefly introduce HLM and then to review some of the considerations that are helpful in answering these questions, including the nature of the data, the model to be tested, and the information desired on the output. Some examples of how the same analysis could be performed in HLM, repeated-measures or mixed ANOVA, and structural equation modeling or path analysis are also provided.
\end{abstract}

Keywords " hierarchical linear modeling; multilevel modeling; repeated-measures; analysis of variance; structural equation modeling; path analysis

Đvhuta@uottawa.ca

\section{Introduction}

Hierarchical linear modeling (HLM) (also referred to as multilevel modeling, mixed modeling, and random coefficient modeling) is a statistical analysis that many researchers are becoming interested in. Previous publications on HLM have provided detailed information on how to perform the analysis (e.g., Raudenbush, Bryk, Cheong, Congdon, \& du Toit, 2011; Woltman, Feldstain, MacKay, \& Rocchi, 2012). Yet there is relatively little information to help researchers decide whether HLM applies to their data and research question, and how to choose between HLM and alternative methods of analyzing the data. The purpose of this tutorial is to review some of the considerations that are helpful in answering these questions. I will focus specifically on the analyses that can be carried out by the software called HLM7 (Raudenbush, Bryk, \& Congdon, 2011).

\section{HLM applies to randomly selected groups}

HLM applies when the observations in a study form groups in some way and the groups are randomly selected (Raudenbush \& Bryk, 2002).

There are various ways of having grouped data. For example, there may be multiple time points per person and multiple persons - these data are grouped because multiple time points are nested within each person. There may be multiple people per organization and multiple organizations, such that people are nested within organizations. There can even be multiple organizations per higher-order group, such as schools nested within cities.
It is possible to have a grouping hierarchy with 2,3 , or 4 levels. An example of a four-level hierarchy is multiple students per school, multiple schools per city, multiple cities per county, and multiple counties - here students are the Level 1 units, schools are the Level 2 units, cities are the Level 3 units, and counties are the Level 4 units. In this tutorial, for the sake of simplicity, I will focus primarily on two-level hierarchies.

As noted above, HLM applies to the situation when the groups are selected at random, i.e., when they represent a random factor rather than a fixed factor. For example, if a study has ten schools (with multiple students in each school), then schools are a random factor if they are randomly selected and the aim is to generalize to the population of all schools; in contrast, schools are a fixed factor if the researcher specifically wanted to draw conclusions about those ten schools, and not about schools in general (and the analysis then becomes an ANOVA).

\section{HLM is an expanded form of regression}

HLM is essentially an expanded form of regression. In most HLM analyses, there is a single dependent variable, though a multivariate option exists as well within the HLM7 software; the dependent variable can be quantitative and normally distributed, or it can be qualitative or non-normally distributed. In this tutorial, I will focus on the case of a single dependent variable that is normally distributed.

Suppose the data set consists of 100 participants, studied at 50 time points each. Roughly speaking, HLM

The Quantitative Methods for Psychology 
] لم: (1)

WHLM: hIm2 MDM File: Sample HLM.mdm

File Basic Settings Other Settings Run Analysis Help

Outcome

Level-1

》 Level-2 《<

INTRCPT2

LEVEL 1 MODEL (bold: group-mean centering; bold italic: grand-mean centering)

T_EXTRAV

S_WBEING $=\pi_{0}+n_{1}($ S_AUTON $)+e$

LEVEL 2 MODEL (bold italic: grand-mean centering)

$\begin{aligned} \pi_{0} & =\varepsilon_{00}+\rho_{01}\left(T_{-} \text {EXTRAV }\right)+r_{0} \\ \pi_{1} & =\varepsilon_{10}+\kappa_{11}\left(T_{-} \text {EXTRAV }\right)+r_{1}\end{aligned}$

Figure 1 - Sample two-level Hierarchical Linear Model.

obtains what is called a Level 1 (or within-group) regression equation for each participant, based on that individual's 50 time points (for a total of 100 equations); the Level 1 equation may have one or more Level 1 independent variables (i.e., independent variables measured at each time point), or it may have no independent variables (the same set of independent variables must be used in all Level 1 equations); the dependent variable must be measured at each time point. Like any regression, the Level 1 equation for a given individual summarizes their data across 50 time points into just a few coefficients: an intercept (which equals the participants' mean score on the dependent variable if the researcher uses what is called group mean centering for each Level 1 independent variable, a common procedure), and a slope for each of the Level 1 independent variables. Each of these coefficients - the intercept and possibly some slopes - then serves as the dependent variable in a Level 2 (or between-group) regression equation; for example, if there are two independent variables in the Level 1 equations, there will be three regression equations at Level 2, one predicting the Level 1 intercept, one predicting the Level 1 slope for one Level 1 independent variable, and the other predicting the slope for the other Level 1 independent variable. Each Level 2 equation has an intercept (which equals the mean intercept or slope across all participants if the researcher uses what is called grand mean centering for each Level 2 independent variable, a common procedure), and it may have one or more Level 2 independent variables (i.e., independent variables measured just once for each participant).

For example, suppose again that there are 100 participants, with 50 time points each, the dependent variable is state well-being (s_wbeing - HLM truncates variable names to eight characters, so you might as well create short names to begin with), the Level 1 independent variable is state autonomy (s_auton), and the Level 2 independent variable is trait extraversion (t_extrav). Below is what the regression equation looks like at Level 1. (Note that e is the error term, indicating that the observed state well-being score at a given time point may differ from the well-being score predicted for that person based on the regression equation; e is always present in the Level 1 equation.)

Participant A's equ.: $\left(s_{\text {wbeing }}\right)=\pi_{0}+\pi_{1}\left(s_{\text {auton }}\right)+e$ Participant B's equ.: $\left(s_{\text {wbeing }}\right)=\pi_{0}+\pi_{1}\left(s_{\text {auton }}\right)+e$ etc.

The intercept $\left(\pi_{0}\right)$ and the slope $\left(\pi_{1}\right)$ values will differ from participant to participant. If state autonomy is group mean centered, the $\pi_{0}$ conveniently equals the mean well-being score across all time points for a given participant, and thus provides an estimate of the participant's trait level of well-being.

Below is what the regression equations look likes at Level 2. (Note that in HLM, you can choose whether or not to include the error term $r_{0}$ and/or $r_{1}$; if the error term $r_{0}$ is included, this implies that the intercept $\pi_{0}$ is assumed to differ from person to person; if the error term $r_{1}$ is included, this implies that the slope $\pi_{1}$ is assumed to differ from person to person.)

$$
\begin{aligned}
& \pi_{0}=\beta_{00}+\beta_{01}\left(\mathrm{t}_{-} \text {extrav }\right)+r_{0} \\
& \pi_{1}=\beta_{10}+\beta_{11}\left(\mathrm{t}_{-} \text {extrav }\right)+r_{1}
\end{aligned}
$$

Figure 1 shows a screen shot of how the model would appear in HLM.

Each equation at Level 2 is a summary across all 100 participants, and each of the four coefficients (those indicated with the letter $\beta$ ) is tested to determine if it differs significantly from zero. If trait extraversion is grand mean centered, $\beta_{00}$ conveniently equals the mean well-being score across all time points and across all participants, called the grand mean, and thus provides an estimate of the average participant's trait level of well-being. The $\beta_{10}$ value provides the average $\pi_{1}$ value across all participants (assuming the Level 2 independent variable(s) is/are grand mean centered). If $\beta_{10}$ is statistically significant, then on average across 
participants, state autonomy significantly relates to state well-being. The $\beta_{01}$ value gives the relationship between trait extraversion and trait well-being (assuming group mean centering was used). Finally, if $\beta_{11}$ is significantly different from zero, this indicates that trait extraversion moderates the strength of the relationship between state autonomy and state wellbeing (this moderation is also called a cross-level interaction, since trait extraversion at Level 2 is interacting with state autonomy at Level 1 ; it is certainly possible to have an interaction between independent variables at the same level, but these are product terms that must be created in the data set before importation into HLM).

\section{When HLM is superior to regular regression}

In the past, before HLM was developed, people simply used a single regular regression for grouped data - either what is called a Level 1 regression or what is called a Level 2 regression. Suppose there are 100 participants and 50 time points per participant, with the variables at each level as discussed before. A Level 1 regression can be used when the researcher is only interested in relationships at Level 1 (e.g.., does state autonomy relate to state well-being), and it involves simply running a regression with a sample size of all 5000 data points as if they came from 5000 independent participants. A Level 2 regression can be used when the researcher is only interested in relationships at Level 2 (e.g., does trait extraversion relate to trait well-being), and it involves running a regression on 100 data points, one per participant, after computing the mean well-being score for each participant.

The question is: When are these regular regressions problematic, making HLM a preferable choice?

\section{When HLM is superior to Level 1 regression - the problem of inflated Type I error}

A Level 1 regression treats data from 100 participants as if it were data from 5000 independent participants. Therein lies the problem. This can lead to a large inflation of Type I error, since the statistical significance of a result depends on sample size. HLM deals with this problem by basing its sample size for inferential statistics on the number of groups (100 in this example), not on the total number of observations (5000 in this example).

The HLM approach has a drawback of its own, as the reader might guess. HLM tends to be on the conservative side when testing relationships at Level 1 , i.e., it has less power than a Level 1 regression would.

There usually is some degree of dependence among the observations from a given group, however, and it is usually advisable to apply an analysis for grouped data, such as HLM. Only if there is no dependence is it appropriate to conduct a Level 1 regression. It is possible to determine the degree of within-group dependence in HLM by testing whether there is variance in the Level 1 intercept across groups - if it does not vary, an analysis for grouped data is not necessary and one can use a Level 1 regression, though one may still want to proceed with an analysis for grouped data on theoretical grounds or for consistency with other analyses that are being conducted. Alternatively, an Intraclass Correlation Coefficient (ICC) smaller than 5\% suggests that an analysis for grouped data is unnecessary (Bliese, 2000). The ICC is the proportion of the total variance in the dependent variable (which is the sum of the between-group variance and the within-group variance) that exists between groups.

\section{When HLM is superior to Level 1 regression - the value of differentiating Levels 1 and 2}

In addition to the reduction of Type I error, there is also a conceptual reason to use HLM instead of Level 1 regression whenever there is a significant variance in coefficients across groups. HLM allows the researcher to separate within-group effects from between-group effects, whereas a Level-1 regression blends them together into a single coefficient.

For example, in one study, I ran an experiencesampling study with about 100 participants and about 50 time points per participant (Huta \& Ryan, 2010). At Level 1, I measured eudaimonia (the pursuit of excellence) and hedonia (the pursuit of pleasure). When I analyzed the data properly, using HLM, I obtained the following results. At Level 1, so at a given moment in time, a person's degree of state eudaimonia and state hedonia correlated negatively, around -.3. Thus, if a person is momentarily striving for excellence, they are probably not simultaneously striving for pleasure. However, at Level 2, so at the trait level, a person's average degree of eudaimonia over the 50 time points and their average degree of hedonia over the 50 time points actually correlated positively, about .30! Thus, if a person often strives for excellence, they also tend to often strive for pleasure. (The latter analysis was performed by choosing one variable, say 


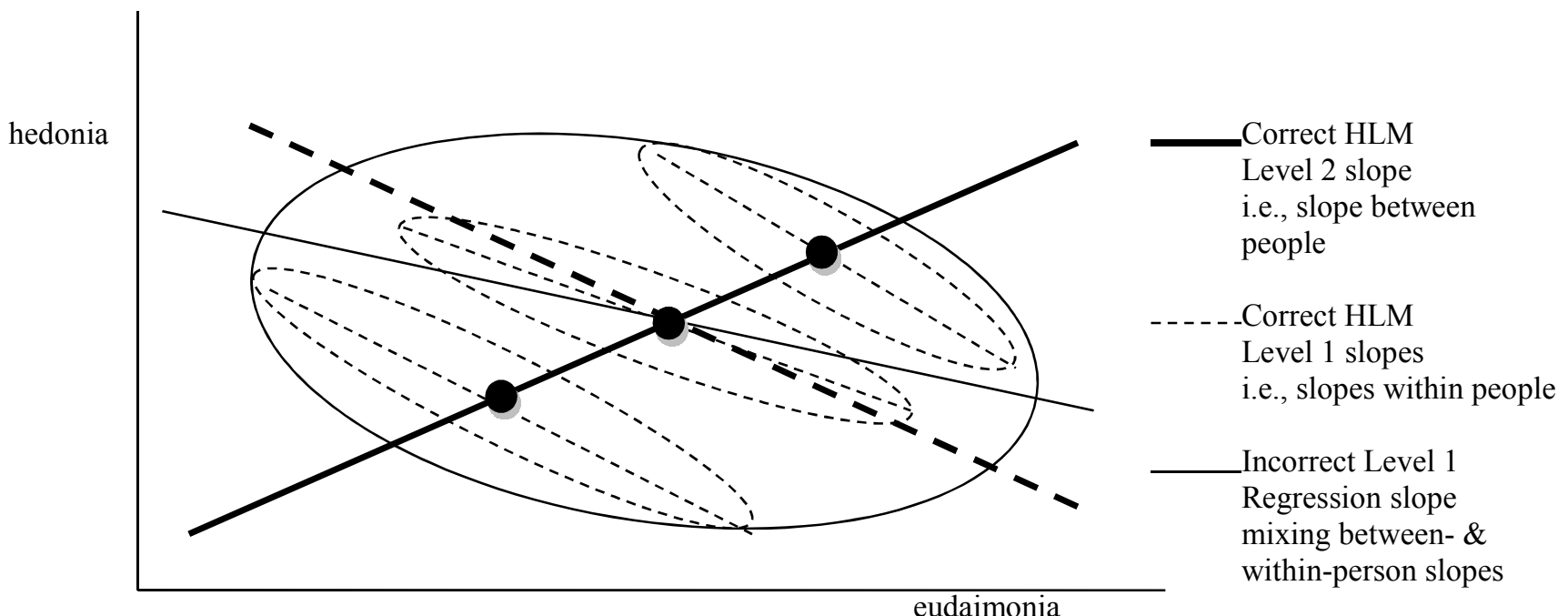

Figure 2 - How two variables can have a negative relationship at the within-group level but a positive relationship at the between-group level in Hierarchical Linear Modeling.

hedonia, as the dependent variable for HLM, and then at Level 2 using each person's mean eudaimonia score as the independent variable, with the mean eudaimonia scores being computed for each participant before running the HLM - in other words, HLM can compute the mean score on the dependent variable for each person, but the mean score on the independent variable has to be computed person by person prior to running HLM). If the data had simply been analyzed using a Level-1 regression, the correlation between eudaimonia and hedonia would be -.10, which is part way between .3 and +.3 and tells us little about the true correlation at Level 1 or at Level 2.

Figure 2 provides an illustration of how the correct slopes obtained through HLM at Levels 1 and 2 can be in opposite directions from each other, and how the Level 1 regression slope is a blend of the two slopes obtained through HLM. Each dotted ellipse represents data across multiple time points for each participants (only three participants are shown). The thin dotted lines running through the dotted ellipses are the lines of best fit for each participant. The thick dotted line is the mean of all the thin dotted lines across participants, and corresponds to the Level 1 or "within-person" correlation of -.3 I obtained using HLM. The thin solid ellipse encompasses all of the data combined across participants, and the thin solid line is the line of best fit through this data and corresponds to the somewhat uninformative correlation of -.1 obtained through a Level 1 regression. The thick solid line corresponds to the Level 2 or "between person" correlation of +.3 obtained using HLM (or using a Level 2 regression), and is the line of best fit through the center points (called centroids) of the ellipses for each participant, which are indicated by large dots.

\section{When HLM is superior to Level 2 regression}

Let me continue with the example of 100 participants and 50 time points per participant, and both eudaimonia and hedonia being measured at each time point. Recall that a Level-2 regression involves taking the mean dependent variable score and the mean independent variable score across the 50 time points for each participant, which produces just 100 observations in total, and then running a regular regression on those means. The results of a Level 2 regression and of HLM will be the same if each participant has the same number of time points and no missing data (and the same variance in the dependent variable). But one lovely feature of HLM is that it allows the researcher to have different numbers of observations per group (i.e., per participant in this example) and furthermore gives greater weight to the groups with more observations (and less variance), which produces slightly more accurate estimates of population values. The greater the differences in sample size (and variance) across groups, the greater the advantage of HLM relative to Level-2 regression. This benefit is subtler and less crucial than the advantage of HLM over Level 1 regression, but it is 

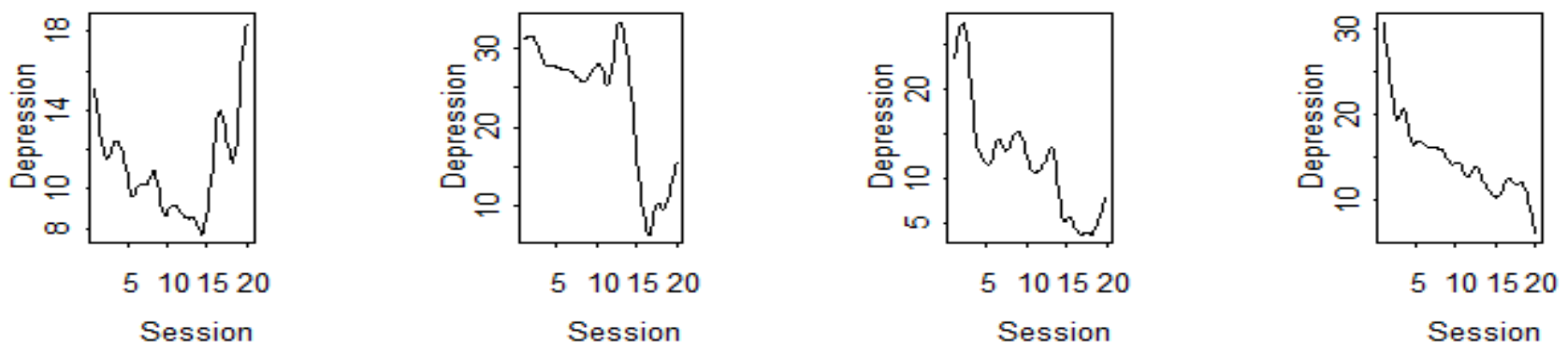

Figure $3 \backsim$ How Functional Data Analysis models the fluctuations a variable undergoes over time.

worth being aware of.

\section{When it is appropriate to use HLM on data from dyads}

When comparing HLM with regular regression, I would like to also make a comment about dyads. Dyads always have two members per group, e.g., husband and wife, caregiver and patient, coach and athlete. It is a common assumption that all research on dyads should be analyzed using HLM. This is often true but not always. HLM only applies when exactly the same set of variables - the dependent variable, or the dependent variable and some Level 1 independent variables - is measured in all members of the group, i.e., in both members of the dyad. For example, HLM applies when the same marital satisfaction questionnaire is given to both the husband and the wife. However, if different variables have been assessed in the two members of the dyad - for example, if the dependent variable assessed in the caregiver is burnout, but the dependent variable assessed in the patient is depression - then HLM does not apply and one would simply run a regular regression (or some other analysis) to predict caregiver burnout, and a separate analysis to predict patient depression.

\section{Different analyses for grouped data}

HLM is not the only method available for dealing with grouped data. The two most common alternatives are structural equation modeling (SEM) (or its simpler version, path analysis), and a general linear model (GLM) with a repeated-measures variable, which I will simply refer to as repeated-measures. Examples of the latter include: mixed design ANOVA/GLM with a repeated-measures/within-subjects variable that is analogous to the dependent variable measured repeatedly at Level 1 in HLM, and one or more between-subjects variables that are analogous to the Level 2 independent variables in HLM; repeatedmeasures ANOVA/GLM with a repeatedmeasures/within-subjects variable; and a pairedsamples t-test, which has only two repeated measures. A less well-known alternative is functional data analysis (FDA), and there are others still, such as growth mixture modeling (GMM).

Let me outline FDA and GMM only briefly, just enough to make the reader aware of these options. I will then discuss repeated measures and SEM (and path analysis) in more detail, describing how the same research question would be addressed with these methods as well as HLM, and listing criteria that can help a researcher choose between HLM, repeated measures, and SEM.

\section{Functional data analysis}

Developed by Ramsay and Silverman (2002, 2005), FDA is used specifically for longitudinal data. It is more flexible than other approaches in that it models the precise pattern of fluctuations that a variable undergoes over time, and every individual/entity can have a different pattern. This makes FDA more flexible than the other approaches I am comparing it with, which assume that a single function (e.g., a straight line, a quadratic curve, a cubic curve, exponential decay) can represent the entire span of data points, and that all individuals can be represented by the same function. For example, Figure 3 shows the pattern of depression scores over the course of therapy for four individuals. A smoothing process can then be applied, to a degree gauged by the researcher, in hopes of eliminating minor fluctuations that are likely to represent random noise, and retaining major fluctuations that are likely to represent a true signal. 
Sample HLM - Level 1.sav [DataSet3] - IBM SPSS Statistics [ File É dit View Data $\underline{\text { Transform }}$ Analyze Direct Mark€

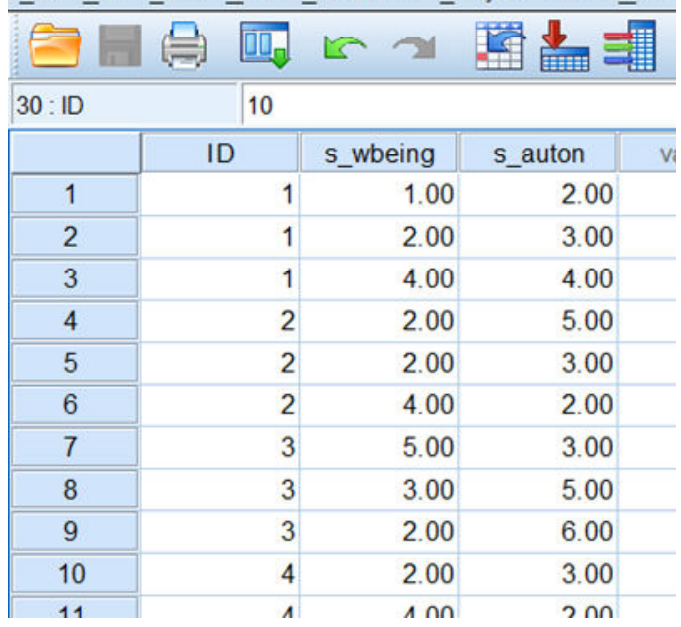

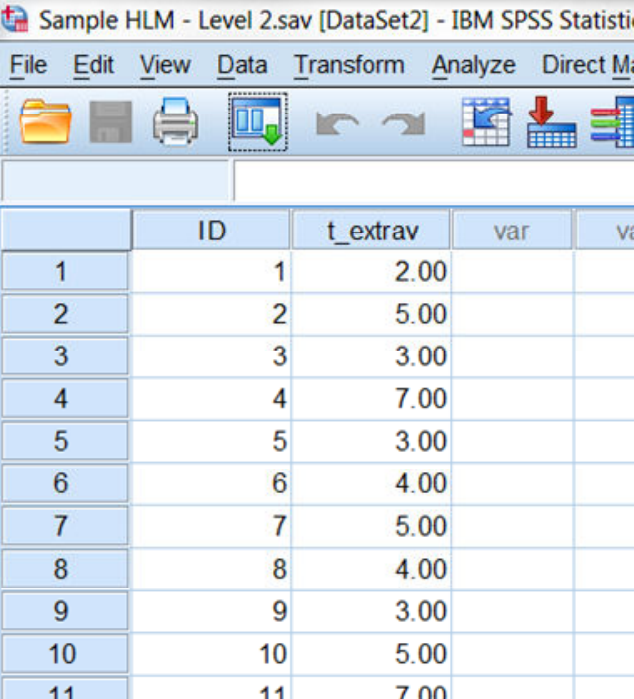

Figure 4 - How to set up the Level 1 and Level 2 data sets for Model A in SPSS prior to using the HLM software for Hierarchical Linear Modeling.

The entire pattern for each individual can then be used in various analyses. For example, analogous to an independent-samples $\mathrm{t}$-test, it is possible to test whether two groups of individuals (such as those in cognitive therapy versus those in behavioral therapy) differ significantly in terms of their mean score at a given therapy session, or even in terms of their slope or rate of improvement (referred to as the velocity of the curve at that time point). Analogous to a regression, it is possible to test whether fluctuations in one variable over time (such as social support) predict later fluctuations in another variable (such as well-being). A principal components analysis can also be performed on the patterns to see at what time points individuals/entities differ most widely from each other - for example, when studying depression scores over the course of therapy, the greatest spread in scores occurs during the last few therapy sessions, since some clients continue to get better while others have trouble with therapy termination and their symptoms get worse.

\section{Growth Mixture Modeling}

Unlike HLM, repeated-measures, SEM, and FDA, which are variable-centered approaches, GMM is a person-centered approach (Jung \& Wickrama, 2008). Variable-centered approaches focus on relationships among variables (another example is factor analysis). Person-centered approaches, such as GMM and cluster analysis, focus on similarities between individuals and aim to classify participants into groups based on their responses across a set of variables. GMM is used for longitudinal data, and it analyzes the trajectories of different individuals to determine whether there are subgroups within which individuals have similar trajectories (Wang \& Bodner, 2007). For example, when analyzing depression scores over the course of therapy, GMM might indicate that there are two groups of individuals - those whose scores progressively improve, and those whose scores remain about the same. Latent class growth analysis is a special case of GMM which assumes that all individuals within a given group have exactly the same trajectory, rather than allowing for variability within groups the way GMM does (Jung \& Wickrama, 2008).

\section{How to set up the same model in HLM, Repeated- measures, and SEM - Model A}

Suppose a two-level data set with three time points per participant has state well-being as the dependent variable at Level 1 , and trait extraversion as the independent variable at Level 2 (suppose there are no Level 1 independent variables). Suppose the researcher wishes to test whether there is a significant link between extraversion and well-being.

Setting up the model in HLM. Prior to importing the data into HLM (i.e., prior to creating the "mdm," the multivariate data matrix), there would be one data set for the Level 1 data (with one line per time point) and a separate data set for the Level 2 data (with one line per participant), as shown in Figure 4 if one is using SPSS (IBM Corp., 2011a). 
WHLM: hlm2 MDM File: Sample HLM.mdm

File Basic Settings Other Settings Run Analysis Help

\begin{tabular}{|c|c|}
\hline Outcome & LEVEL 1 MODEL (bold: group-mean centering: bold italic: grand-mean centering) \\
\hline Level-1 & S WBFING = +0 \\
\hline$\gg$ Level-2 く< & O_VVOLI \\
\hline INTRCPT2 & LEVEL 2 MODEL (bold italic: grand-mean centering) \\
\hline T_EXTRAV & $n_{0}=\varepsilon_{00}+\varepsilon_{01}\left(T_{-}\right.$EXTRAV $)+r_{0}$ \\
\hline
\end{tabular}

Figure 5 - How to set up the analysis for Model A using the HLM software for Hierarchical Linear Modeling.

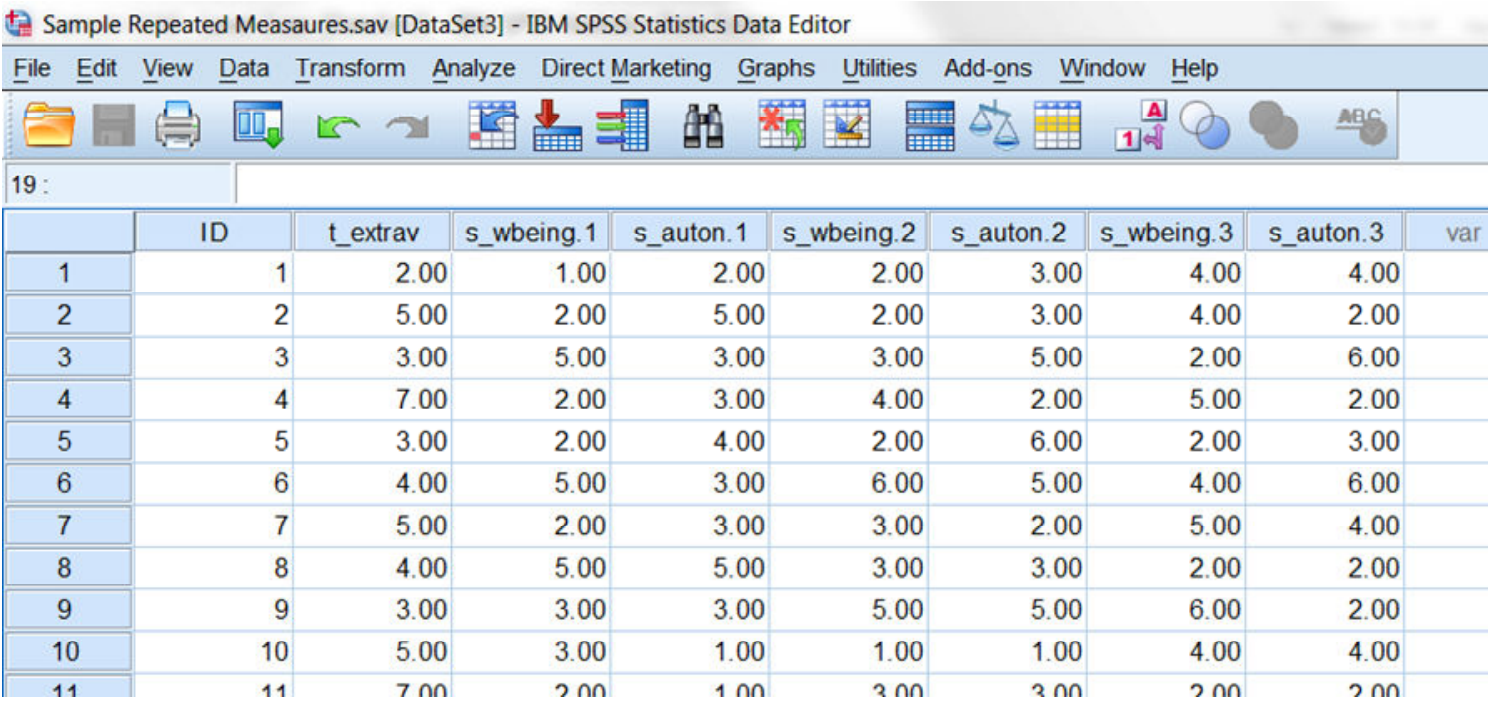

Figure 6 - How to set up the data set for Models A and B in SPSS prior to Repeated-measures

In HLM, the analysis would then be set up as shown in Figure 5. (The error term $\mathrm{u}_{0}$ is kept in the model, indicating that the intercept of state well-being is assumed to show some variance from person to person even after controlling for the role of trait extraversion, a reasonable assumption to start out with until there is evidence to the contrary.)

On the output, to determine whether extraversion relates to well-being, one would see whether the coefficient $\beta_{01}$ is statistically significant.

Setting up the model in repeated measures. For repeated measures, there would simply be one data set (with one line per participant), as shown in Figure 6 if one is using SPSS (Lacroix \& Giguère, 2006).

The three variables in the data set that represent state well-being at the three time points would then be used to create the within-subjects factor/variable (which might be called "time_point" and which one would designate as having 3 levels), and the one variable in the data set that represents trait extraversion would be the between-subjects covariate.
In other words, if one were using SPSS, the analysis would be run as shown in Figure 7 (for guidelines on how to perform various repeated-measures models, see Tabachnick \& Fidell, 2007).

On the output, to determine whether extraversion relates to well-being, one would see whether the test of the between-subjects effect for extraversion was statistically significant.

Setting up the model in SEM. Prior to importing the data into an SEM software such as AMOS (IBM Corp., 2011b), the data would again be set up as shown in Figure 6 - in other words, there would be one line per participant/group (for guidelines on running SEM, see Arbuckle, 2011; Kline, 1998). (I focus here on AMOS because of its wide use and availability, especially since it has become associated with SPSS, though other software such as LISREL and EQS are used often in the multilevel case).

The model would then be set up as shown in Figure 8 if one is using AMOS. Technically, Figure 8 is a path analysis rather than a structural equation model, given 


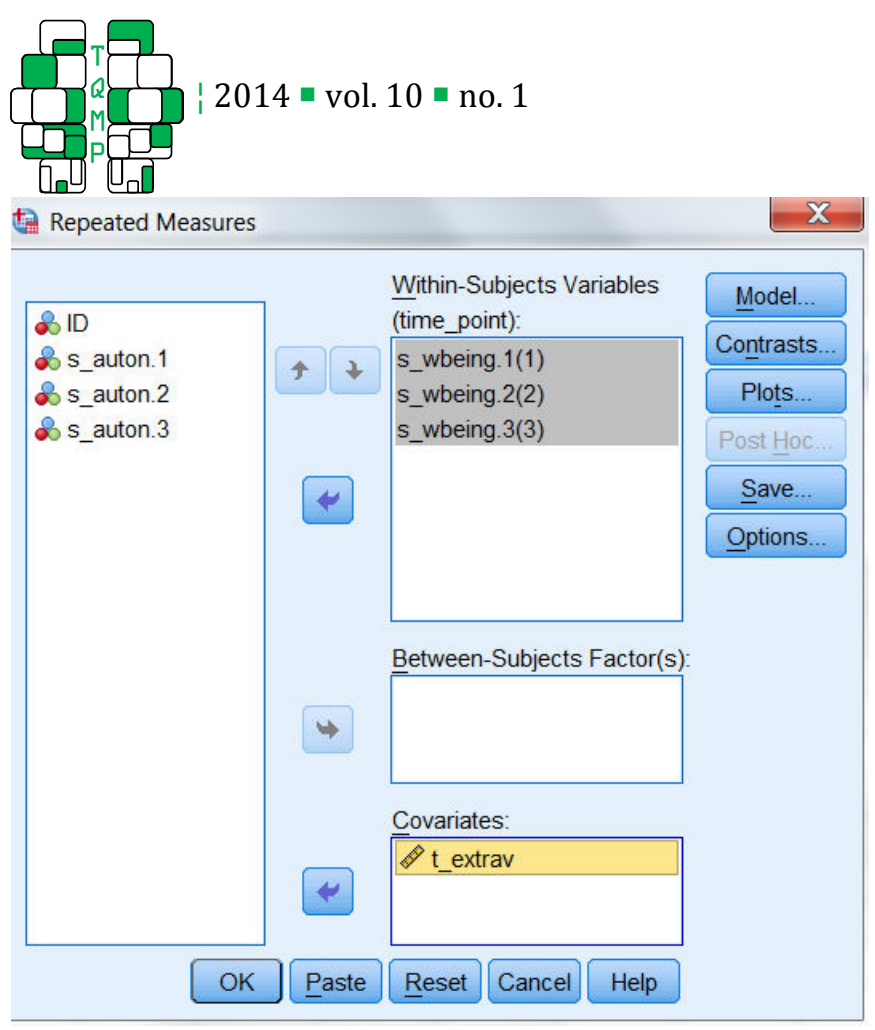

Figure 7 - How to set up the analysis for Model A using SPSS for Repeated-measures analysis.

that it examines the relationships between measured variables (indicated with rectangles), and not between latent variables which are factors extracted from multiple measured variables (which would be indicated with ellipses). Notice that all three regression coefficients are constrained to be equal, since they have all been assigned the same name "a," so that the analysis parallels HLM where a single value is obtained for the link between extraversion and well-being; similarly, all of the error terms have received the same label "e1." Alternatively, the researcher may allow the regression coefficients and error terms to vary, to see if the Level 2 independent variable has a different impact on well-being at each time point, a feature not available in HLM or repeated-measures.

On the output, to determine whether extraversion relates to well-being, one would see whether the regression coefficient "a" was statistically significant.

\section{How to set up the same model in HLM, Repeated- measures, and SEM - Model B}

Now suppose a two-level data set with three time points per participant has state well-being as the dependent variable at Level 1 , and suppose the researcher wishes to test whether there is a linear increasing trend in well-being over time.

Setting up the model in HLM. Prior to importing the data into HLM, one would need to set up a variable called "time" in the Level 1 data set, and assign it the values 1 ,

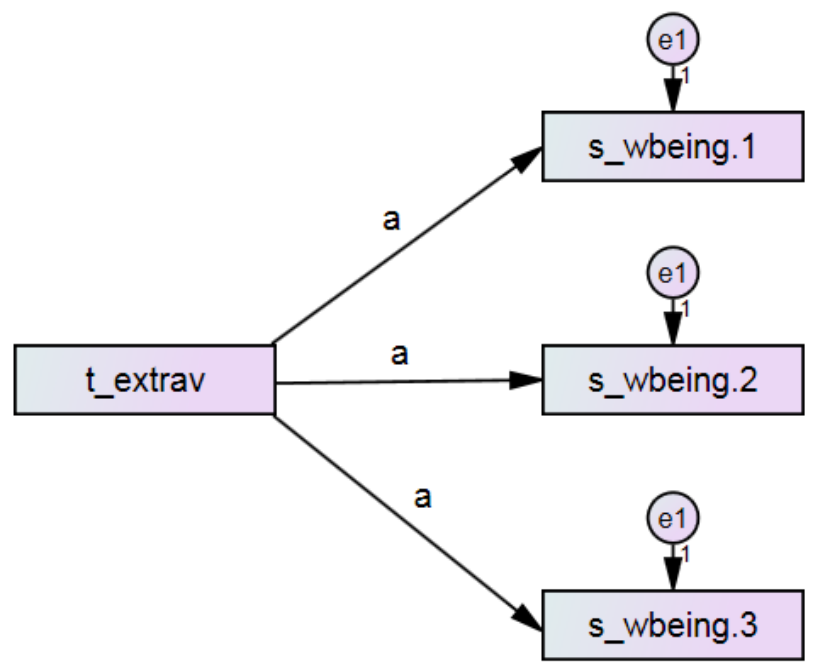

Figure 8 - How to set up the analysis for Model A using AMOS for Path Analysis.

2, and 3 for time points 1, 2, and 3 (assuming they were equally spaced), as shown in Figure 9. The Level 2 data set is also shown in Figure 9 (and is the same as in Figure 4).

In HLM, the analysis would then be set up as shown in Figure 10, if one assumes the intercept and slope will vary from person to person (a good assumption to begin with, until there is evidence to the contrary).

On the output, to determine whether there is a linear increasing trend over time, one would see whether the coefficient $\beta_{10}$ is positive and statistically significant. In other words, one would see whether there is a relationship between time and state wellbeing, on average across participants.

Setting up the model in repeated measures. For repeated measures, the data set in Figure 6 would be used as is.

The three variables that represent state well-being would then be used to create the within-subjects variable, as shown in Figure 11.

On the output, to determine whether there is a linear trend over time, one would see whether the within-subjects contrast for time_point is statistically significant. One would also need to check the descriptive statistics or a plot, to see if the linear trend was indeed increasing rather than decreasing.

Setting up the model in SEM. For SEM, the data structure shown in Figure 6 would be used as is.

When using the AMOS software to run the SEM, the model would be set up as shown in Figure 12 (which is 


\begin{tabular}{|c|r|r|r|r|r|}
\hline \\
\hline
\end{tabular}

\begin{tabular}{l} 
Sample HLM - Level 2.sav [DataSet2] - IBM SPSS Statisti \\
\hline File Edit View \\
\hline
\end{tabular}

Figure 9 " How to set up the Level 1 and Level 2 data sets for Model B in SPSS prior to using the HLM Software for Hierarchical Linear Modeling.

called a latent difference score analysis - e.g., Hawley, Ho, Zuroff, \& Blatt, 2007; McArdle, 2001). The terms in ellipses would be created by the researcher, and the two paths from "Sn" would be constrained to be equal, by being assigned the same coefficient "a." (The " $\mathrm{d}$ " values are the latent and error-free variables assumed to produce the measured variables; the "delta d" values are the differences in the " $\mathrm{d}$ " values from one time point to the next, being equal to the later time point minus the earlier time point, thus the term latent difference score analysis; "Sn" is the latent slope assumed to underlie the "delta d" values; and the regression coefficients "a" are fixed to be equal - typically all set to 1 - to reflect the expectation that change over time will be linear for each participant.)

On the output, to determine whether there is an increasing linear trend over time, one would see whether the coefficient "a" is positive and statistically significant.

\section{Choosing between HLM, Repeated-measures, and SEM}

Let me now review a variety of criteria that come into play when choosing a method for analyzing grouped data. The focus will be on HLM, repeated-measures, and SEM, as these are the most commonly used methods. Sometimes more than one method is possible, but I will emphasize the situations where one method is preferable to another.

In reading the criteria below, it will become clear that I was quite selective in creating Models A and B above to compare across HLM, repeated measures, and SEM. Only certain models and data sets can be analyzed using all three methods. It will also become clear that choosing an acceptable method is a complex process, with many considerations to take into account. Some considerations are rigid and can entirely rule out a method, whereas other considerations are more flexible and the ultimate selection of method will rely on the judgment of the researcher.

\section{When the hierarchy has three or more levels}

Hierarchies with three or more levels are typically analyzed using HLM, though if sample sizes are small at all the lower levels of a hierarchy, it may also be feasible to use SEM. Repeated-measures only applies to two-level hierarchies.

\section{When sample size differs from group to group}

Of the three analyses, only HLM can be used when sample size differs from group to group at any of the lower levels of a hierarchy.

\section{When there is missing data at Level 1}

Missing data at Level 1 can be handled by HLM, which can simply work with the data it is given, if the researcher chooses the "delete when running analyses" option when importing data into HLM. (There is also an option to delete an entire Level 2 group if it has any missing data at Level 1 , if the researcher chooses the "delete when making mdm" option). SEM requires that 


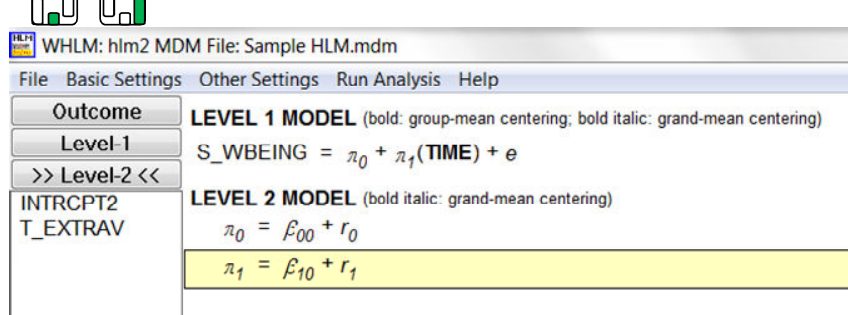

Figure 10 - How to set up the analysis for Model B using the HLM Software for Hierarchical Linear Modeling.

all data be present at all levels of the hierarchy, and can impute missing data as a part of the analysis. Repeatedmeasures deletes the entire Level 2 group if it has any data missing at Level 1.

\section{When there is missing data at a higher level of the hierarchy}

When there is missing data for a group (e.g., for a participant) at a higher level of a hierarchy, HLM deletes all information regarding that group at the level of the hierarchy where the data is missing and also at all lower levels of the hierarchy. Thus, one should consider performing data imputation at the higher level where the data is missing before proceeding to HLM. As noted above, SEM requires that all data be present at all levels of the hierarchy, and can impute missing data as a part of the analysis. Repeated-measures deletes the entire Level 2 group if it has any data missing at Level 2.

\section{When group members are distinguishable}

Observations at a lower level of the hierarchy are either distinguishable or indistinguishable. When observations are distinguishable, they can be ordered in some kind of consistent non-arbitrary way - for example, when the first observation in each group represents "time 1," the second observation represents "time 2," and so on; or when the first observation in each group represents "husbands," and the second observation represents "wives." When observations are indistinguishable, they do not fall in any natural order for example, when the researcher is simply studying multiple organizations with multiple employees within each organization, and there is no sequence to the employees, so that employee 1 in organization $A$ does not correspond in any way to employee 1 in organization $\mathrm{B}$.

HLM can be used whether observations at the lower levels are distinguishable or indistinguishable, though if observations at a given level are distinguishable, the researcher typically includes an index variable in the

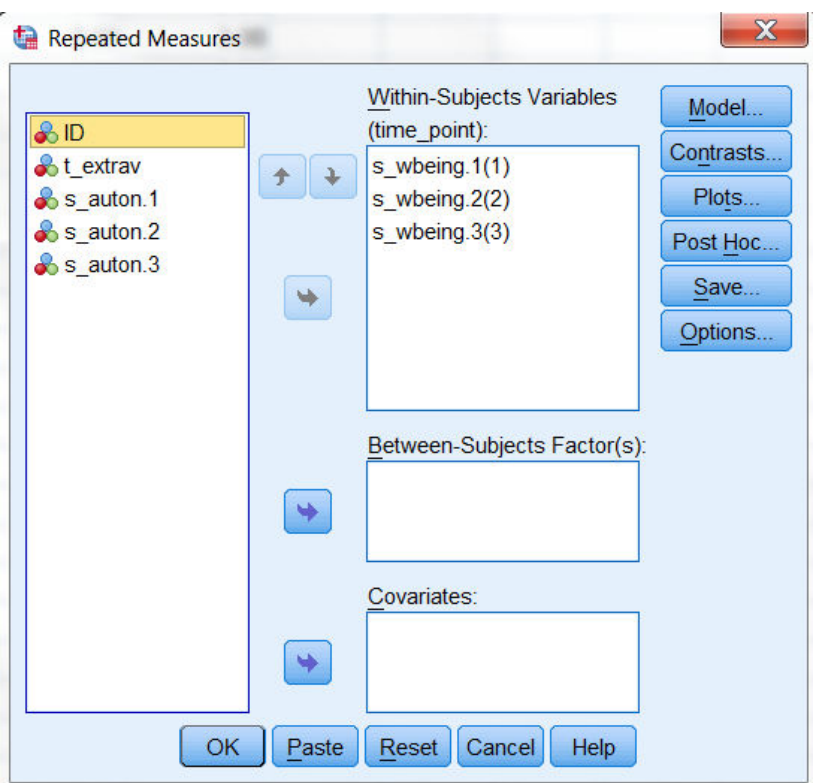

Figure 11 - How to set up the analysis for Model B using SPSS for Repeated-measures analysis.

analysis which represents the sequence of observations (e.g., a variable called "time" with the values 1, 2, 3, etc.).

SEM is primarily used when observations are distinguishable, though there is a somewhat complicated procedure that can be used for indistinguishable observations.

Repeated-measures is primarily used when observations are distinguishable, though the multivariate alternative to repeated-measures can be used when observations are indistinguishable.

\section{When there are independent variables at a lower level of the hierarchy}

In SEM, there is no absolute limit on the number of independent variables that can be incorporated into a lower level of the hierarchy (though an overly complex model can raise a number of difficulties, such as insufficient degrees of freedom, and poor fit of fit indices that penalize model complexity).

In repeated-measures, the only Level 1 independent variable that is "built into" the analysis is time or repeated measure, and additional independent variables cannot be added.

In HLM, the researcher needs to be careful about the number of independent variables include at a lower level. At a given level, as in any regression where one wishes to carry out inferential statistics, HLM requires more degrees of freedom than there are coefficients to 


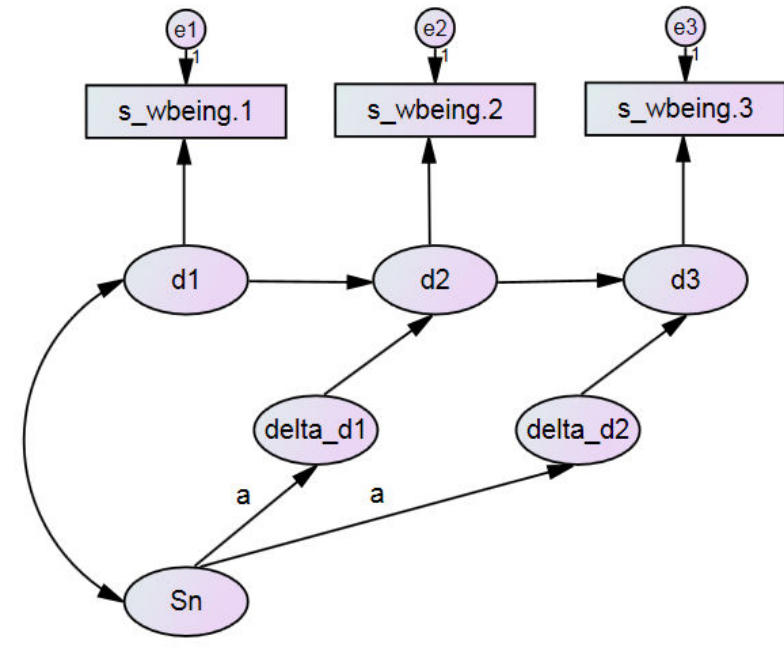

Figure 12 " How to set up the analysis for Model B using AMOS for Path Analysis.

be estimated (in at least some of the groups, though not necessarily all). Consider the Level 1 equation below:

$$
\begin{aligned}
(\text { state well-being }) & =\pi_{0}+\pi_{1}(\text { state autonomy }) \\
& +\pi_{2}(\text { state mindfulness })+e
\end{aligned}
$$

There are three coefficients in this equation - the intercept $\pi_{0}$ and the two slopes $\pi_{1}$ and $\pi_{2}$ - and therefore a minimum of four observations is required. Another way of summarizing this is as follows: if there are $\mathrm{K}$ independent variables, HLM requires $\mathrm{K}+2$ observations. Thus, if a given level of the hierarchy consists of dyads, there are only two observations per group and there is no room for independent variables at that level!

When there are too few observations for the number of independent variables at Level 1, some researchers artificially increase the number of observations (e.g., Maguire, 1999) by treating individual items or subscales of items on the dependent variable measure as if they were separate measurements of the dependent variable. For example, if a researcher has dyads at Level 1 and the dependent variable is a four-item scale, the researcher could treat each of the four items as if it were a separate observation of the dependent variable, which would artificially boost the sample size at Level 1 to eight observations per dyad. Alternatively, if a researcher has dyads at Level 1, the dependent variable is an 18-item scale, and one wants to boost the sample size to six observations per dyad, one can create three parallel subscales of the dependent variable (as highly intercorrelated as possible), and treat each of them as if it were a separate measurement of the dependent variable. Researchers may vary in their comfort level with this practice of boosting the number of measurements of the dependent variable, but the practice is more acceptable to the degree that the items or subscales of the dependent variable measure the same concept - if their content is diverse, the practice is harder to justify. Naturally, the strategy is only possible if the dependent variable is a multi-item scale.

\section{When there are independent variables at the highest level of the hierarchy}

All three analysis - HLM, repeated-measures, and SEM can handle any number of independent variables at the highest level of the hierarchy (within reason - the number of independent variables should not take up a large fraction of the degrees of freedom at that level, and with too many independent variables, one starts to run into the problem of over-fitting, i.e., the equation starts modeling a lot of the noise rather than primarily the signal in the data).

\section{When one wishes to test cross-level interactions}

Suppose a researcher wishes to test a cross-level interaction, such that trait extraversion moderates the effect of state autonomy on state well-being. This can easily be tested in HLM, and the software itself will create the interaction term, the researcher does not need to create it in the data beforehand. SEM can also test a cross-level interaction, but the researcher needs to create all the interaction terms in the data set before analysis (unless the higher level independent variable is a qualitative one, such as gender, in which case the SEM model can be run once for each gender, and differing results for the two genders indicate an interaction), and SEM quickly begins to consume many degrees of freedom as the number of observations at the lower level increases. Both HLM and SEM can test interactions between two independent variables in non-adjacent levels of a hierarchy, such as Levels 1 and 3 , as well as interactions between independent variables at three or more levels (though SEM is likely to consume even more degrees of freedom than for two-way interactions). In Repeated-measures, the only cross-level interaction possible is one between the repeated measure variable at Level 1 (e.g., time_point) and a between-subjects variable at Level 2 (e.g., t_extrav).

In repeated-measures, since the only independent variable possible at Level 1 is time or repeated measure, the only cross-level interaction possible is an

The Quantitative Methods for Psychology 

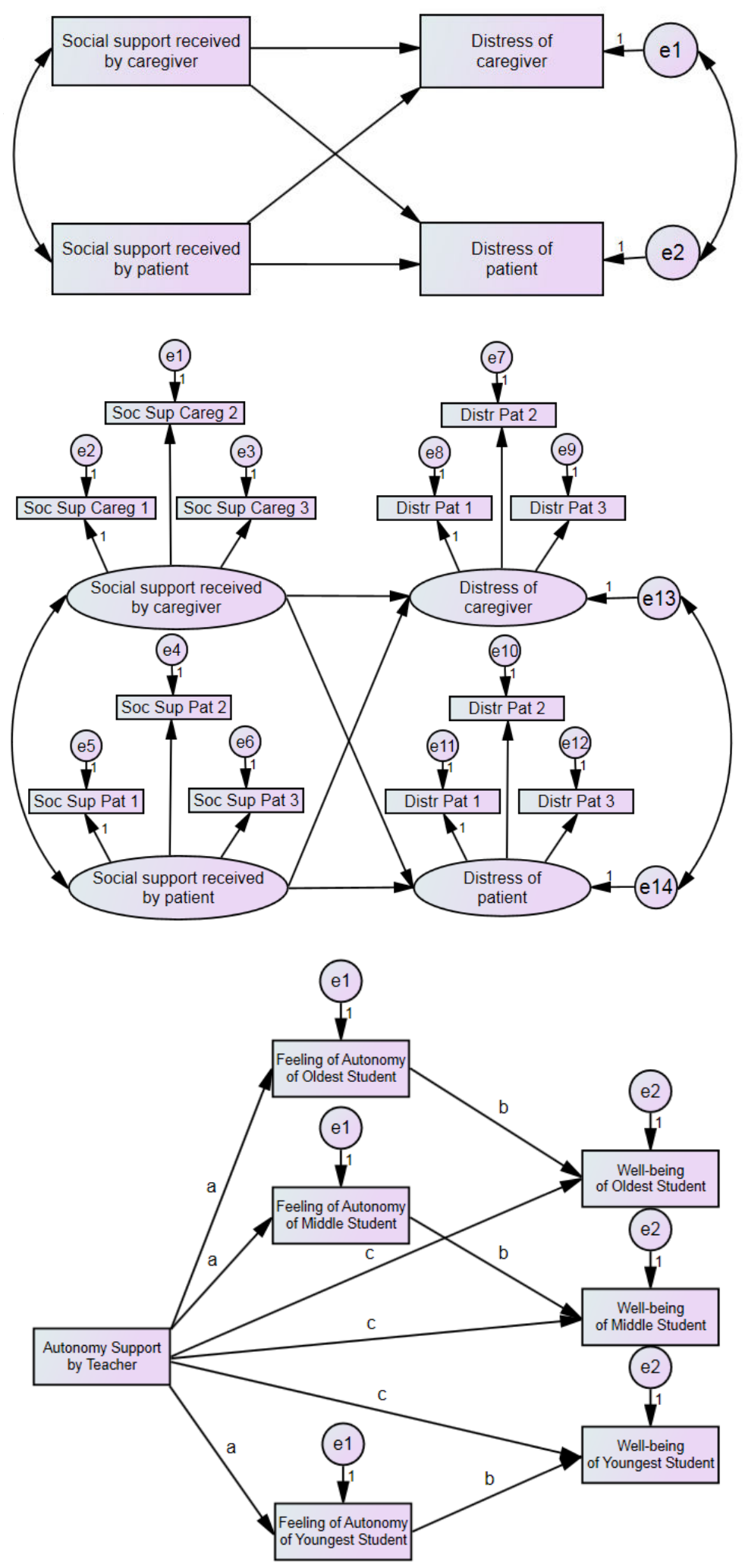

Figure 13 - Examples of models that are more complex than a single regression, and which are therefore frequently best suited for structural equation modeling or path analysis.

\section{When one wishes to test same-level interactions}

Both HLM and SEM can test interactions at the same level, but the interaction terms must be created in the data before analysis. Repeatedmeasures can only test interactions at Level 2, since it cannot have multiple independent variables at Level 1.

\section{When the model does not have a multiple regression structure}

Both HLM and repeated-measures require a multiple regression structure, such that one or more independent variable(s), and possibly some interactions between them, all directly predict a single dependent variable (or a composite of dependent variables). For any model more complex than a multiple regression, SEM is most often used. For example, SEM is appropriate when a variable in the model predicts more than one other variable, or when a variable serves both as an outcome and a predictor. Figure 13 shows examples of structures that are more complex than a single multiple regression (the first model is a path analysis of what is called an actor-partner interdependence model, which is commonly used in dyad research, and in this case the focus is only on relationships between variables at Level 1 - the individual person - rather than Level 2 - the patientcaregiver dyad; the second model is the structural equation modeling version of the first model, where each concept in an ellipse is a factor extracted from three measured variables; the third model is a path analysis of a mediation where the independent variable is at Level 2 and the mediator and outcome are at Level 1, and where the regression coefficients and error terms could also be allowed to vary).

HLM can be used to analyze some complex models, but the model must first be broken down into pieces that each have a regression structure, with each piece analyzed separately. Furthermore, in a given piece of the model, the predictors need to be at the same level of the hierarchy as the outcome or at a higher level of the hierarchy, but not at a lower level; this restriction does not apply to 
SEM.

The set of complex models that repeated-measures could apply to is extremely restricted. It would again require that the model be broken into pieces with a regression structure. Furthermore, the last regression in a causal chain could only have Level 2 variables predicting a Level 1 outcome (unless there is one predictor and it is time point or repeated measure), and all preceding steps in the causal chain would be analyzed using regular regressions with Level 2 variables predicting a Level 2 outcome.

\section{When the sample size is too small (or too large)}

Restrictions in sample size at one or more levels of the hierarchy may also push the researcher away from certain analysis options. Ideally, one would use a power analysis software to determine the sample size required at each level to achieve adequate power. (One software applicable to HLM is Optimal Design, which is available for free on the internet, at http://sitemaker.umich.edu/group-based/optimal_ design_software; one software that is applicable to repeated-measures is $\mathrm{G}^{*}$ Power, also available for free on the internet, at http://www.psycho.uniduesseldorf.de/abteilungen/aap/gpower3/downloadand-register; power analysis for SEM is less well established.) Nevertheless, there are some general guidelines that can help a person determine whether a sample size may be adequate for HLM, repeatedmeasures, and/or SEM.

Sample size required at each level in HLM. At a lower level of the hierarchy, HLM can work with as few as two observations per group if there are no independent variables at that level, or as few as $\mathrm{K}+2$ observations if there are $\mathrm{K}$ independent variables, as noted earlier. Many papers have been published with just two, three, or four observations per group, so these sample sizes are quite common. To reach $80 \%$ power, however, group sizes often need to be 15 or greater (though 5 or 10 is sometimes sufficient). To be able to reliably report the regression equations of individual groups (which is done only occasionally, e.g., when there are multiple students per school and each school would like to know the results for that particular school), the required sample size is often closer to 50 .

At the highest level of the hierarchy, having an adequate sample size is more critical, since it is the sample size at this level which influences the power of the analyses. In other words, the number of groups at the highest level needs to be large enough for the results to be generalizable to the population of all groups. Some publications have had Level 2 sample sizes as small as 10 or 20, and in some research areas (e.g., work with animals or brain scans) this is difficult to avoid. To reach $80 \%$ power, however, sample sizes upward of 60 are typically required.

Sample size required at each level in repeated-measures. At Level 1, repeated-measures can work with any sample size of 2 or greater.

At Level 2, to reach $80 \%$ power, sample sizes above 60 are often required.

Sample size required at each level in SEM. At a lower level of the hierarchy, SEM can have as few as 2 observations per group, and does not require that there be $\mathrm{K}+2$ observations for $\mathrm{K}$ independent variables, as noted earlier. If there are too many observations, the SEM model will use too many degrees of freedom unless corresponding regression coefficients are constrained to be equal and corresponding error terms are constrained to be equal (as shown in the third model of Figure 14).

At the highest level of the hierarchy, SEM typically requires a larger sample size than does HLM or repeated-measures. Recommendations vary widely, but most people would agree that 100 is a bare minimum, and the more common recommendation is 200 or more; alternatively, many people use Bentler and Chou's (1987) suggestion that there be at least 5 observations per free parameter.

\section{When the researcher seeks certain information in the output}

Thus far I have discussed considerations that relate to the nature of the data or the nature of the model to be tested, when choosing between HLM, repeatedmeasures, and SEM. These analyses also differ in the information that appears on the output, so I will now review some of these differences. I will not provide an exhaustive list of the differences, I will only focus on one major difference that is commonly of interest: HLM provides information based on how coefficients differ across groups (i.e., how coefficients at one level of the hierarchy differ across units of a higher level of the hierarchy), whereas repeated-measures and SEM provide information about how coefficients differ across repeated measures.

A separate regression equation for each group. Only HLM provides a separate regression equation for each higher-level group - for example, if there are multiple organizations and multiple employees within each 
organization, HLM can provide the regression equation across employees within a given organization. (This is an additional option that must be requested when running the analysis, and the coefficients of the equations can be obtained from an SPSS file generated with the name "resfil.") HLM actually provides several versions of these regression equations - the Ordinary Least Squares (OLS) version simply provides the coefficients that would be obtained if a regular regression were run on the data within a given group; an Empirical Bayes version provides coefficients for a given group that are influenced by the average coefficients when summarizing across all groups, to the degree that the data for the given group are unreliable, i.e., based on a small sample size and/or highly variable; and a more fine-tuned Conditional Empirical Bayes version, which provides coefficients for a given group that are influenced by coefficients in groups that have similar characteristics to the group in question.

Reliability of a coefficient to determine whether the regression equation for each group can be reported reliably. Only HLM provides an estimate of the average reliability, across groups, of each lower level coefficient. Among other things, this reliability informs the researcher whether the regression equations of individual groups can be reported (the reliability ranges from 0 to 1 , and values in the neighborhood of .80 suggest that individual regressions can be reported).

Variance of a coefficient across groups. Only HLM reports the variance of each coefficient across groups, and tests the significance of this variance (e.g., for a three-level hierarchy, the output indicates how much Level 1 coefficients vary across Level 2 units, how much Level 1 coefficients vary across Level 3 units, how much Level 2 coefficients vary across Level 3 units, and how much cross-level interactions between Level 1 and 2 independent variables vary across Level 3 units). Among other things, a significant variance in a coefficient across units of a higher level provides statistical justification for adding independent variables at the higher level in hopes of accounting for some of that variance.

Correlations between coefficients across groups. Only HLM provides the correlations between lower level coefficients across groups. For example, if a research has a two-level hierarchy where the dependent variable is depression severity and the Level 1 independent variable is time, a negative correlation between the Level 1 intercept and slope indicates that the more severe the depression, the faster/steeper the decrease in depression over time.

A separate regression coefficient for each repeated measure. If a researcher wishes to know whether the slope of the relationship between a predictor and outcome differs across repeated measurements of that predictor and outcome, the researcher would require SEM. For example, in the third diagram of Figure 14, a researcher may wish to determine whether the link between a feeling of autonomy and well-being differs for the oldest, youngest, and middle student - in that case, the researcher would simply remove the letter " $b$ " from each regression arrow and thereby allow the coefficient to vary.

SEM can also be used to determine whether a higher-level independent variable relates differently to the dependent variable for each repeated measure. For example, in Figure 14, this would be accomplished by removing the constraint "a" on the link between teacher autonomy support and each student's feeling of autonomy, thereby allowing the strength of the link to vary. Repeated-measures can similarly show how the link between a Level 2 independent variable and the dependent variable differs across repeated measures, if one requests the parameter estimates option.

Variance of the dependent variable across repeated measures. If a researcher wishes to know whether the mean score on the dependent variable varies across repeated measurements, they would use repeatedmeasures (and leave out any Level 2 independent variables). The between-subjects effect for the intercept would indicate whether this variance is significant.

\section{Summary}

In sum, Hierarchical linear modeling (HLM) applies to data that are grouped in some way, such as: multiple cities, with multiple schools within each city, and multiple students within each school. In this example, cities would be referred to as Level 3 of the hierarchy, schools would be referred to as Level 2, and students would be referred to as Level 1. In addition, HLM applies when the groups (e.g., cities and schools) are randomly selected, such that the researcher's aim is to generalize to the results to the population of all groups.

The HLM method is an expanded form of regression, whereby a separate regression is obtained within each group, and the dependent variable is always measured at the lowest level of the hierarchy. The coefficients (intercept and slopes) from the within-group 
regressions then serve as dependent variables in several regressions at the between-group level. In this way, all of the main effects and interactions a researcher might be interested in can be determined: the main effect of a within-group independent variable, the main effect of a between-group independent variable, the interaction between independent variables at the same level of the hierarchy, and the interaction between independent variables at different levels of the hierarchy.

Analysis using HLM (or another method used for grouped data) preserves the multi-level nature of the data, and thus has several advantages over a single regression performed on the data. The greatest advantage is that a grouped analysis protects the researcher against inflated Type I error.

In addition to HLM, methods that sometimes apply to grouped data include repeated-measures analyses (such as mixed design ANOVA/GLM) and structural equation modeling (SEM) or its simpler version path analysis (and possibly even functional data analysis, Growth mixture modeling or Latent Class Growth Analysis, or other options). This paper provided two examples of models that could be set up in HLM, repeated-measures, and SEM/path analysis, and outlines how the data set and analysis for each method would be set up.

This paper then provides considerations that can help a researcher in choosing between HLM, repeatedmeasures, and SEM/path analysis if they have grouped data. These considerations include: the number of levels in the hierarchy, sample size, missing data, distinguishability of group members, the number of independent variables, the nature of the interactions to be tested, whether the model to be tested has a regression structure, and the information one desires on the output (e.g., whether one is more interested in differences between groups or differences between repeated measures).

Together, the information in this paper sheds some light on a frequently neglected topic: how a researcher can decide whether HLM applies to their data and research question, and how a researcher can choose between HLM and alternative methods of analyzing such data.

\section{References}

Arbuckle J. L. (2011). AMOS 20.0 user's guide. Crawfordville, FL: Amos Development Corporation. Bentler, P. M., \& Chou, C. P. (1987) Practical issues in structural modeling. Sociological Methods \& Research, 16, 78-117.

Bliese, P. D. (2000). Within-group agreement, nonindependence, and reliability: implications for data aggregation and analysis. In K. J. Klein, \& S. W. J. Kozlowski (Eds.), Multi-level theory, research and methods in organizations: Foundations, extensions, and new directions (pp. 349-381). San Francisco, CA: Jossey-Bass.

Hawley, L. L., Ho, M. R., Zuroff, D. C., \& Blatt, S. J. (2007). Stress reactivity following brief treatment for depression: Differential effects of psychotherapy and medication. Journal of Consulting and Clinical Psychology, 75, 244-256.

Huta, V., \& Ryan, R. M. (2010). Pursuing pleasure or virtue: The differential and overlapping well-being benefits of hedonic and eudaimonic motives. Journal of Happiness Studies, 11, 735-762.

IBM Corp. (2011a). IBM SPSS Statistics for Windows, Version 20.0. Armonk, NY: IBM Corp.

IBM Corp. (2011b). IBM SPSS AMOS, Version 20.0. Armonk, NY: IBM Corp.

Jung, T., \& Wickrama, K. A. S. (2008). An introduction to Latent Class Growth Analysis and Growth mixture modeling. Social and Personality Psychology Compass, 2, 302-317.

Kline, R. B. (1998). Principles and practice of structural equation modeling. New York, NY: Guilford.

Lacroix, G. L., \& Giguère, G. (2006). Formatting data files for repeated-measures analyses in SPSS: Using the Aggregate and Restructure procedures. Tutorials in Quantitative Methods for Psychology, 2, 20-25.

Maguire, M.C. (1999). Treating the dyad as the unit of analysis: A primer on three analysis approaches. Journal of Marriage and the Family, 61, 213-223.

McArdle, J. J. (2001). A latent difference score approach to longitudinal dynamic analysis. In R.

Cudeck, S. Du Toit, \& D. Sörbum (Eds.), Structural equation modeling: Present and future (pp. 341380). Lincolnwood, IL: Scientific Software International.

Ramsay, J. O., \& Silverman, B. W. (2002). Applied functional data analysis: Methods and case studies. New York: Springer.

Ramsay, J. O., \& Silverman, B. W. (2005). Functional data analysis, Second Edition. New York: Springer.

Raudenbush, S. W., \& Bryk, A. S. (2002). Hierarchical Linear Models: Applications and data analysis methods, Second Edition. Newbury Park, CA: Sage. 
Raudenbush, S. W., Bryk, A. S., Cheong, Y. F., Congdon, R. T., \& du Toit, M. (2011). HLM7: Hierarchical Linear and Nonlinear Modeling. Chicago, IL: Scientific Software International.

Raudenbush, S. W., Bryk, A. S., \& Congdon, R. (2011). HLM7 for Windows [Computer software]. Skokie, IL: Scientific Software International, Inc.

Tabachnick, G. G., and Fidell, L. S. (2007). Experimental designs using ANOVA. Belmont, CA: Duxbury.
Wang, M., \& Bodner, T. E. (2007). Growth mixture modeling: Identifying and predicting unobserved subpopulations with longitudinal data. Organizational Research Methods, 10, 635-656.

Woltman, H., Feldstain, A., MacKay, J. C., \& Rocchi, M. (2012). An introduction to hierarchical linear modeling. Tutorials in Quantitative Methods for Psychology, 8, 52-69.

\section{Citation}

Huta, V. (2014). When to Use Hierarchical Linear Modelling. The Quantitative Methods for Psychology, 10 (1), $13-28$.

Copyright (C) 2014 Huta. This is an open-access article distributed under the terms of the Creative Commons Attribution License (CC BY). The use, distribution or reproduction in other forums is permitted, provided the original author(s) or licensor are credited and that the original publication in this journal is cited, in accordance with accepted academic practice. No use, distribution or reproduction is permitted which does not comply with these terms. 